\title{
Defining Eligibility for Services Under PL 99-457
}

\author{
JACK P. SHONKOFF \\ University of Massachusetts Medical School
}

SAMUIEL J. MEISELS

University of Michigan

The concepts of multivariate risk and mandated family focus are relevant considerations for states formulating eligibility criteria. This article examines categories of eligibility and their relation to these two factors. An alternative viewpoint on eligibility is presented that challenges professionals to rethink both the purposes of screening and evaluation, as well as the relation between primary health care and community-based early intervention programs.

One of the central issues left to the states by the framers of the Part $\mathrm{H}$ statute and regulations under Public Law 99-457 concerns the definition of who is eligible for services. The law mandates that two groups of infants and toddlers be served-those with established diagnoses and those with demonstrated developmental delays. In contrast, decisions regarding service provision for young children who are considered to be at risk for significant future developmental problems have been left to the discretion of individual states. Clearly, the breadth or narrowness of the definitions adopted for these categories will have a major influence on their cost and im. pact. Thus, it is essential that determination of eligibility be informed by knowledge about the development and adaptation of vulnerable children and their families and by an understanding of the relatively modest predictability of both infant assessments in general and risk categories in particular.

Two issues are especially relevant to considerations of eligibility: (1) the concept of the multiple risk model and (2) the mandated family focus of the law (Shonkoff $\mathcal{E}$ Meisels, 1990). In the first case, research in child development demonstrates that outcomes for children at risk for disabilities cannot be predicted reliably on the basis of single risk factors. Rather, predictions must be informed by an understanding of the multidimensional, transactional nature of the developmental process over time (Kochanek, Kabacoff, \& Lipsitt, 1990). With regard to the second issue, Part $\mathrm{H}$ mandates that all services be guided by an Individualized Family Service Plan (IFSP) that is developed jointly by parents and service providers in order to assure that intervention goals respond to the needs of both children and families (Krauss, 1990). The implications of these two issues for determining service eligibility criteria deserve brief elaboration.

\section{THE MUILTIPLE RISK MODEL}

We now know that the majority of children who fall within many of the traditionally ac- 
cepted categories of biological or environmental risk (e.g., those who were born prematurely or those who are raised by young, unmarried mothers with low incomes) will develop normally. Thus, if the presence of a single biological or environmental risk factor were to be accepted as a sufficient criterion for eligibility for comprehensive services, early intervention systems would enroll large numbers of children and families who do not necessarily need those services. Nevertheless, when compared to the general population, a greater proportion of children who fall within these categories of increased vulnerability will develop significant developmental difficulties. Therefore, if mechanisms were developed to monitor vulnerable children, and if the level and pattern of service delivery for each were based on an individualized assessment of the balance among risk and protective factors within both the child and the family, resource allocation could be better targeted to those who are at greatest risk. How one develops practical eligibility criteria for a statewide system that conforms to these principles is the challenge before us (Meisels E Wasik, 1990).

\section{THE MANDATED FAMILY FOCUS OF THE $L A W$}

The key to meeting this challenge lies in the Part $\mathrm{H}$ mandate for IFSPs. A thoughtful implementation of this mandate should lead to a highly differentiated service system that can provide a broad range of service options, extending from ongoing monitoring and the provision of educational materials to intensive, multidisciplinary team efforts. If the technical skills of a number of highly trained professionals are required, services will be more costly. When such relatively simple interventions as developmental surveillance and parental access to information are sufficient, services are less expensive but no less worthy of the label "early intervention." This approach is consistent with the definition of early intervention as a continuum of individualized services ranging from periodic assessment to the intensive mobilization of highly specialized therapeutic and educational resources. Such an approach makes it possible to think of eligibility in a more flexible context, based upon the following three categories: established conditions, developmental delay, and documented risk (biological or environmental).

\section{CATEGORIES OF ELIGIBILITY}

\section{Established Conditions}

This category includes children with established diagnoses that invariably result in disability or developmental delay (for example, chromosomal disorders, such as Down syndrome or fragile-X syndrome; inborn errors of metabolism, such as Tay-Sachs disease or Hurler syndrome; other genetic disorders, such as tuberous sclerosis; cerebral palsy and other neuromuscular disorders; disorders secondary to congenital infections, such as symptomatic cytomegalovirus, toxoplasmosis, and human immunodeficiency virus; severe attachment disorders, such as autism; sensory disorders; and disorders secondary to exposure to toxic substances, such as intrauterine alcohol and cocaine). In such cases, though an evaluation of both child and family is necessary for the purpose of developing an Individualized Family Service Plan, no further evaluation is required for the purpose of establishing eligibility.

\section{Developmental Delay}

This category includes children with significant delays or atypical patterns in their devel- 
opment, with or without a specific diagnosis or identified etiology. As for those with established conditions, an evaluation is necessary in order to design an IFSP, but a confirmation of delayed development based on a valid and reliable diagnostic assessment should be sufficient to confirm eligibility for services.

In considering the criteria for a determination of developmental delay, some further explanation about eligibility is needed. For example, in many states, eligibility is based on psychometric criteria of very questionable validity. In some cases, children are provided services only if their developmental abilities are shown to be $1,1 / 2$, or 2 standard deviations below the mean on a developmental assessment. However, tests may differ in their standard deviations, or their standardization samples may be so different from each other that a comparison of two similar standard deviations or even two similar means would be meaningless.

Another approach used widely for determining eligibility is based on a comparison between a child's performance on a developmental scale and the expected performance for a child of the same age. Eligibility is then linked to a prescribed discrepancy or percentage delay between performance level and chronological age. However, very few states that use percentage delays for eligibility determination differentiate the amount of delay according to age, even though a $25 \%$ delay in a 1-year-old's development is quite different from a $25 \%$ delay in a 3-year-old's. This approach assumes not only that the available developmental scales are sufficiently accurate to make such fine distinctions, but that they are equivalent across the age span, both assumptions that are highly questionable. Thus, rather than relying solely on standard deviations or percentage delays, a more appropriate approach would be a functional one that ascertains a child's and family's current abilities and resources and uses this additional information to interpret the clinical significance, not just the statistical significance, of test scores.

\section{Documented Biological Risk}

This category includes children whose pediatric history or current circumstances reveal significant biological condition(s) that do not lead invariably to developmental delay or disorder, but that carry a greater probability of delay and disability than is found in the general population (for example, low birth weight, asymptomatic congenital cytomegalovirus infection, perinatal asphyxia, chronic lung disease, failure to thrive). Infants and toddlers who fall within this group require a comprehensive child and family evaluation to determine their eligibility for services on a case-by-case basis. Individual service plans for such children and their families will be quite diverse and may include varying combinations of phone monitoring, information and referral, periodic reassessment, brief home visits, participation in activity centers, parent support groups, intensive home or center-based intervention, individual parent counseling, or specialized therapeutic interventions.

\section{Documented Environmental Risk}

This category includes children whose pediatric history, caregiving circumstance, and current family situation contain risk factors that do not lead invariably to developmental delay, but that carry a greater probability of delay or disability than is found in the general population (for example, maternal mental illness, parental substance abuse, significant family social disorganization, extreme poverty, parental intellectual impairment, disturbed parent-child interaction, low maternal education, family isolation and lack of support, homelessness, history of inade- 
quate prenatal care, child abuse, or neglect). Those who fall within this group also require a comprehensive child and family evaluation to determine service eligibility. If that evaluation confirms that the child's developmental course is likely to be different from that of a child without such environmental risk condition(s), then a determination should be made about the type and level of services for which the child and family are eligible, within the continuum of services described above.

As noted earlier, the essential characteristic of the last two categories is the fact that a significant proportion of children who meet the criteria for biological or environmental risk are likely to do well developmentally, with or without early intervention services. The identification of those who are at greatest risk of having significant developmental problems - those who should have the highest priority for service delivery-can only be addressed effectively through serial screenings and selective diagnostic evaluations conducted over an extended period of time. In other words, the nature and extent of participation in the early intervention system must be guided by the findings of individualized assessments, rather than by arbitrary categorical distinctions. Such an approach will assure both a prudent use of scarce public resources and the provision of appropriate services for families with children at highest risk for significant problems.

\section{RETHINKING THE CONCEPT- ELIGIBILITY FOR WHAT?}

Stated simply, the target population for early intervention must be thought of in terms of eligibility for assessment and ongoing formulation of an appropriate service plan-not in terms of eligibility for a fixed set of comprehensive services. Within such a framework, exclusion of appropriate service recipients would be minimized and a rational allocation of resources would be facilitated. The gatekeeper function would no longer be fulfilled by a single test, metric, or criterion. Such a single factor approach is inconsistent with both the multidimensional definition of early intervention described in Part $\mathrm{H}$ and the multidetermined nature of risk and disability (Meisels \& Provence, 1989). Thus, a family whose child has an established diagnosis and whose internal family resources and external support networks are strong might be served best by the simple provision of educational materials, periodic reevaluation, and comprehensive pediatric care. Another family, whose personal resources for nurturing a young child are extremely limited and whose support network is highly tenuous, may have a relatively healthy infant whose need for extensive prevention services is great.

Valid and reliable screening and subsequent formal evaluation of children who fall within any of the four eligibility categories could be delivered through existing early intervention programs or by regional assess. ment teams that meet standards set by each state. Such evaluation services should be designed to serve as a link between pediatric primary care settings and community-based early intervention programs, thereby facilitating both easy access into the early intervention system and ongoing coordination of necessary health, developmental, and support services. In the final analysis, service plans must be tailored to individual needs and must be determined jointly by the family and the potential service providers. Thus, the portal of entry into the early intervention system should focus on eligibility for an evalua. tion and for the negotiation of an Individualized Family Service Plan, rather than on automatic eligibility for a comprehensive, predefined array of services. 


\section{REFERENCES}

Kochanek, T.T., Kabacoff, R.I., \& Lipsitt, L.P. (1990). Early identification of developmentally disabled and at-risk preschool children. Exceptional Children, 56, 528-538.

Krauss, M.W. (1990). New precedent in family policy: Individualized Family Service Plan. Exceptional Children, 56, 388-395.

Meisels, S.J., \& Provence, S. (1989). Screening and assessment: Guidelines for identifying young disabled and developmentally vulnerable chil. dren and their families. Washington, DC: National Center for Clinical Infant Programs.
Meisels, S.J., \& Wasik, B.A. (1990). Who should be served? Identifying children in need of early intervention. In S.J. Meisels \& J.P. Shonkoff (Eds.), Handbook of early childhood interven. tion (pp. 605-633). New York: Cambridge University Press.

Shonkoff, J.P., \& Meisels, S.J. (1990). Early childhood intervention: The evolution of a concept. In S.J. Meisels \& J.P. Shonkoff (Eds.), Hand. book of early childhood intervention (pp. 3-32). New York: Cambridge University Press.

Address correspondence to: Dr. Jack Shonkoff, University of Massachusetts Medical School, 55 Lake Avenue North, Worcester, MA 01655 